NASA CONTRACTOR REPOR T

0
0
$\vdots$
$\simeq$
$\vdots$
$\Sigma$

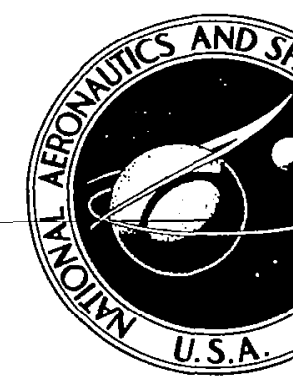

EXPERIMENTS IN

LOW ENERGY PLASMAS

by E. H. Holt and J. H. Noon

\author{
Prepared by \\ RENSSELAER POLYTECHNIC INSTITUTE \\ Troy, N. Y. \\ for
}

NATIONAL AERONAUTICS AND SPACE ADMINISTRATION - WASHINGTON, D. C. - OCTOBER 1967 


\section{EXPERIMENTS IN LOW ENERGY PLASMAS}

By E. H. Holt and J. H. Noon

Distribution of this report is provided in the interest of information exchange. Responsibility for the contents resides in the author or organization that prepared it.

Issued by Originator as Technical Report No. 67-1

Prepared under Grant No. NsG-48 by

RENSSELAER POLYTECHNIC INSTITUTE

Troy, N.Y.

for

NATIONAL AERONAUTICS AND SPACE ADMINISTRATION

For sale by the Clearinghouse for Federal Scientific and Technical Information Springfield, Virginia 22151 - CFSTI price $\$ 3.00$ 



\section{CONTENTS}

SUMMARY

INIRODUCTION 2

MICROWAVE STUDIES OF ELECTRON ENEERGY LOSS

FROCESSES IN AFTERGLOW PLASMAS

Radiation 'Temperature Measurements

Electron Collision Probability

Non-Maxwellian Electron Velocity Distribution

1

3

4

$\frac{5}{6}$

ELECTROMAGIETIC WAVE PROPAGATION THROUGH MAGNETOPIASMAS THEORY

The Transformation Matrix

Propagation along the Magnetic Field

The Characteristic Polarization

The Polarization for Maximum Transmission

EIECTROMAGNETIC WAVE PROPAGATION THROUGH MAGNETOPLASMAS EXPERIMENT

Free Space Propagation

14

Guided Wave Propagation

14

Microwave Iens Systems

19

20

PLASMA STABILITY AND :TRANSPORT IN MAGNETIC FIELDS 20

Analysis of the Modes of the Helical Instability
of the Helium Positive Column

Subthreshold Excitation 22

Minimum B Geometry 24

Cesium Plasma Cell 24

The Initial Value Problem of Plasma Transport
Phenomena

$\begin{array}{ll}\text { Diffusion } & 27\end{array}$

ATTEMPT TO GENERATP PLASMA OSCIITATIONS WITH A

RELATIVISTIC ELECTRON BEAM 29

REFHERENCES 


\section{EXXERIMENTIS IN LOW ENERGY PLASMAS}

Microwave Studies of Electron Energy Loss Processes in Afterglow Plasmas

Electromagnetic Wave Propagation through Magnetoplasmas

Plasma Transport and Stability in Magnetic Ficlds

E. H. Holt and J. H. Noon

Rensselaer Polytechnic Institute, Troy, New York

\section{SUMMARY}

This report reviews research carried out in the Plasma Research Laboratory (renamed the Plasma Dynamics Laboratory in August, 1966) at Rensselaer Polytechnic Institute under NASA grant no. NsG 48. The work is described under three major headings, microwave studies of electron energy loss processes in afterglow plasmas, electromagnetic wave propagation through magnetoplasmas and studies of plasma transport and stability in the presence of magnetic fields. 
The results are reported of a comprehensive research program on the properties of collision dominated plasmas. Cross section data, transport coefficients (including the special case of time-dependent transport), propagation coefficients for electromagnetic waves and plasma oscillations are discussed. The current convective instability is studied in transverse fields and a strong stabilizing effect is reported.

Understanding of the behavior of collision dominated plasmas is important in ionospheric work and in certain types of plasma accelerator. Because they are comparatively well understood such plasmas provide a favorable starting point for studies of plasma stability and containment in various magnetic field configurations.

The work reported here includes a detailed study of the approach to thermodynamic equilibrium of a nitrogen afterglow plasma. Microwave interferometry provides an account of the variation of the plasma density and microwave radiometry adds important information about the changing energy of the electron gas. Finally, a time resolved measurement of the velocity distribution of the electrons is made in order to provide a complete description of the electron behavior.

A theoretical formulation is made of the effect of the wave polarization on the propagation of electromagnetic waves in magnetoplasmas. An experimental demonstration of the theory is provided using microwaves propagating in free space. The use of the measurement of wave polarizations as a diagnostic tool is enhanced by the use of guided waves. A plasma cell with turnstile junctions suitable for such studies is described.

Collision dominated plasmas in strong magnetic fields are subject to the helical instability. Measurements of the oscillations which accompany this instability have resulted in a definitive understanding of the modes of the instability. A pre-excitation technique has provided a check of the linear theory which avoids the non-linear character of the f'ull grown instability. The effects of transverse magnetic fields have been examined and it has been found that a minimum $B$ field has a strong stabilizing effect.

A microscopic theory of time-dependent transport phenomena is described. The advantages of this approach compared to modified macroscopic approach are discussed. 
In order to monitor the electron temperature of the plasma as a function of time a gated microwave radiometer was developed (Ref. 1). The radiometer has a time resolution of 1 microsecond and a temperature sensitivity of around $10^{\circ} \mathrm{Kelvin}$ when measuring temperatures near room temperature.

Two special waveguide cells were developed for microwave studies of afterglow plasmas (Refs. 2 and 3). These bakeable metal cells have advantages over glass discharge vessels and reduce uncertainties in the boundary conditions to be applied.

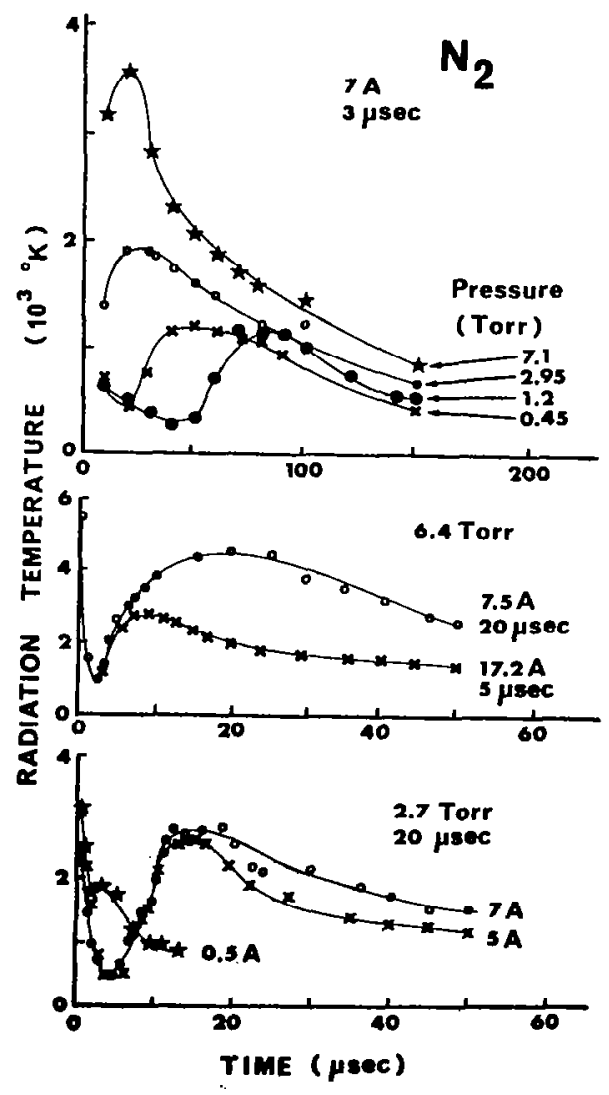

Figure 1. Radiation temperatures measured for different gas pressures and dif'f'erent energy input conditions in the active discharge. Pulse widths from 3 microseconds to 20 microseconds and discharge currents from 0.5 to 17.5 amps were used. 
Radiation Temperature Measurements

Using the gated microwave radiometer, noise radiation from the electrons of the plasma was monitored as a function of time both in the active discharge and in the afterglow period following termination of the discharge. Radiation temperatures were obtained by comparison with a standard noise source, as described in Reference 1.

For the nitrogen afterglow the initial fall.in the radiation temperature after termination of the active discharge agrees with what would be expected due to electron collisions with the neutral molecules. However, the radiation temperature then rises with time, reaching a maximum and then falling off slowly. Typical results for different pressures and different discharge conditions are shown in Figure 1. It is obvious that a source of energetic electrons must exist in the afterglow and that the energy input in the active discharge determines the magnitude of the effect. This work has been reported as a NASA Technical Note (Ref. 4) and as an article in the Physical Review (Ref. 5).

The effect of a small proportion of $\mathrm{CO}_{2}$ on the radiation temperature of the nitrogen plasma is quite striking, see Figure 2. There is a near resonant energy match between the $\mathrm{v}=1$ vibrational level of the $\mathrm{N}_{2}$ molecule and the $\left(00^{\circ}\right.$ l) rotational level of the $\mathrm{CO}_{2}$ molecule leading to strong quenching of the $\mathrm{N}^{*}(\mathrm{v}=1)$ level (Refs. 9 and 10). Thus, it is likely that excited molecules play an important role in the afterglow.

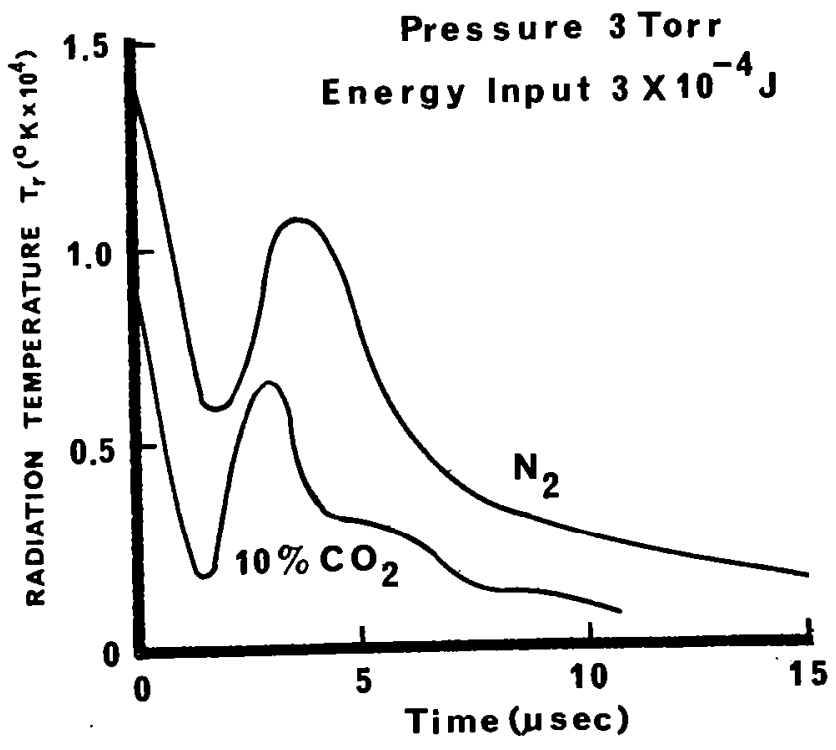

Figure 2. Changes in radiation temperature of the nitrogen afterglow caused by admixture of a small percentage of $\mathrm{CO}_{2}$ in the discharge. 
Calculations by other workers (Refs. 11 and 12) of the probability for momentum transfer $\mathrm{P}_{\mathrm{c}}$ of low energy electrons ( $<\mathrm{leV}$ ) in nitrogen as a function of energy, based on microwave conductivity measurements, have not included direct measurement of the electron temperature in the plasma. Results obtained by waveguide transmission (Ref.. 11) and resonant cavity (Ref. 12) techniques differ by a factor of five at low energies. Although the measurements were carried out at different times in the afterglow, both sets of calculations included the assumption of a Maxwellian distribution of electron velocities whether or not the mean electron energy is greater than that of the neutrals.

Our data indicates that calculations of $P_{c}$ based on the assumption that only 50 microseconds are required for the electrons in the nitrogen afterglow plasma to reach the temperature of the neutral gas is questionable. This point was raised by Formato and Gilardini (Ref. 13) who observed a slow relaxation of electron temperature in nitrogen but did not carry out measurements at times close to the termination of the active discharge.

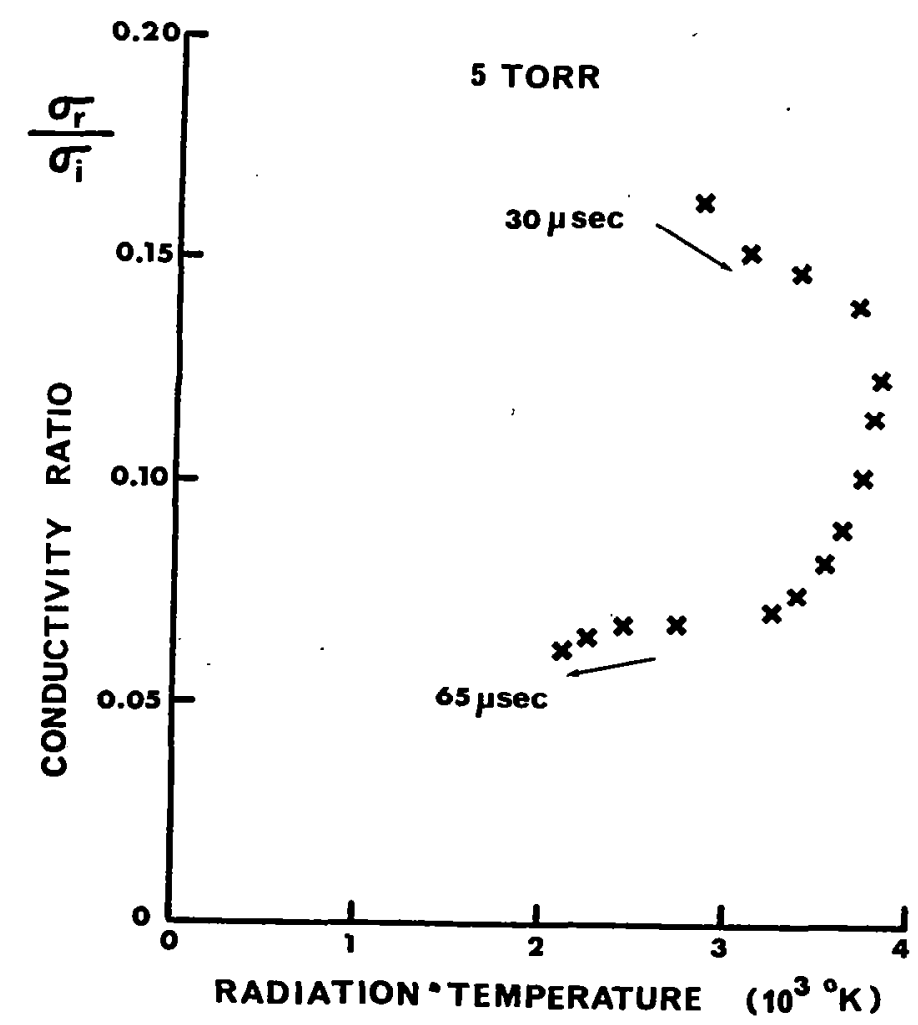

Figure 3. Ratio of real and imaginary parts of the microwave conductivity $\left(\sigma_{r} / \sigma_{i}\right)$ plotted as a function of radiation temperature for post-discharge times ranging from 30 to 65 microseconds. This should fall off monotonically but does not do so, indicating that the calculations based on an assumed Maxwellian distribution are incorrect. 
We have calculated $P_{c}$ as a function of energy by equating the measured radiation temperature to the electron temperature. Although the results were generally close to those derived from transport coefficients (Ref. 14) and resonant cavity measurements (Ref. 12) they showed a wide scatter. Furthermore investigation of the behavior of the conductivity ratio $\sigma_{r} / \sigma_{i}$, (Figure 3 ), reveals that it is a multi-valued function of the radiation temperature instead of decreasing monotonically. A calculation shows that electron-ion collisions cannot explain the effect at these temperatures (approximately $2000^{\circ} \mathrm{K}$ ). The most likely explanation is that the radiation temperature cannot be equated to the electron temperature and that the form of the electron velocity distribution is not Maxwellian in the early post-discharge period, as assumed in the calculations. This work has been reported in a NASA Technical Note (Ref. 15) and a Physical Review article (Ref. 5).

Non-Maxwellian Electron Velocity Distribution

We have applied a microwave technique first described by Fields et. al. (Ref. 16) to determine the electron velocity distribution in the nitrogen afterglow plasma from the noise emission spectrum. If a longitudinal magnetic field is applied, measurement of the noise power from the plasma, as a function of magnetic field, for a range of field strength such that the electron cyclotron frequency is close to the microwave observational frequency, may be analyzed to determine departures from a Maxwellian distribution. A magnetic field of around 3,250 gauss is required for measurements at $X$-band frequencies. The experimenta 1

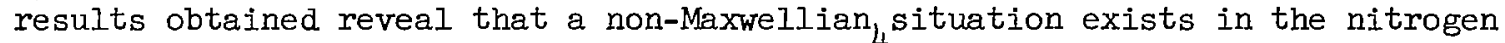
afterglow for periods of time of more than $10^{4}$ electron-neutral mean free times and that the form of the distribution function changes rapidly following termination of the active discharge.

The radiation temperature $T_{r}$ is related to the form of the electron velocity distribution function $f(v)$ for the case when the plasma is immersed in a magnetic field by

$$
T_{r}=-\frac{1}{k} \frac{\int_{0}^{\infty} \frac{v(v)}{v^{2}(v)+\left(\omega-\omega_{b}\right)^{2}}}{\int_{0}^{\infty} \frac{v(v)}{v^{2}(v)+\left(w-\omega_{b}\right)^{2}} \frac{\partial f(v) v^{4} d v}{\partial u}}
$$

where $\mathrm{k}$ is the Boltzmann constant, $\mathrm{v}$ the electron velocity, $v(\mathrm{v})$ the electron collision frequency with neutral particles, $u$ the electron energy, $w$ the radiation frequency and $\omega_{\mathrm{b}}$ the electron cyclotron frequency.

In order to use this expression it is necessary to assume a form for $f(v)$. The Maxwellian velocity distribution is of the form $f(v) \sim \exp \left(-\mathrm{mv}^{2} / 2 \mathrm{kT}\right)$ so that as a simple form of a more general distribution function we will use 


$$
\mathrm{f}(\mathrm{v}) \sim \exp (-\mathrm{bv} a)
$$

We then find that $b$ is related to the average energy $\langle u\rangle$ by the expression

$$
b=\left[\frac{1}{2}<\frac{m}{u}>\frac{\Gamma(5 / a)}{\Gamma(3 / a)}\right]^{a / 2}
$$

where $\Gamma(5 / \boldsymbol{a}), \Gamma(3 / \boldsymbol{a})$ are non-integer Gamma functions. The difference between various forms of the distribution function is illustrated in Figure 4.

For nitrogen the electron-neutral collision frequency $v(v)$ is an increasing function of $v$ over almost all electron energies of interest. When $T_{r}$ is calculated it is found to peak at $\omega_{b}=\omega$ for a distribution represented by $\alpha>2$ remain constant for $a=2$, and show a dip at $\omega=\omega$ for $a<2$.

Figure 5 shows how the exponential parameter $a$ and the average energy $\langle u\rangle$ changes as a function of time in the nitrogen afterglow. These results were obtained by matching the experimental results against the theoretical curves. The time required for the parameter a to return to a value of 2 , corresponding to a Maxwellian distribution is over 20 microseconds, corresponding to more than $10^{4}$ electron-neutral mean free times. The electron temperature is still an order of magnitude higher than that of the neutral gas when a Maxwellian distribution is reached.

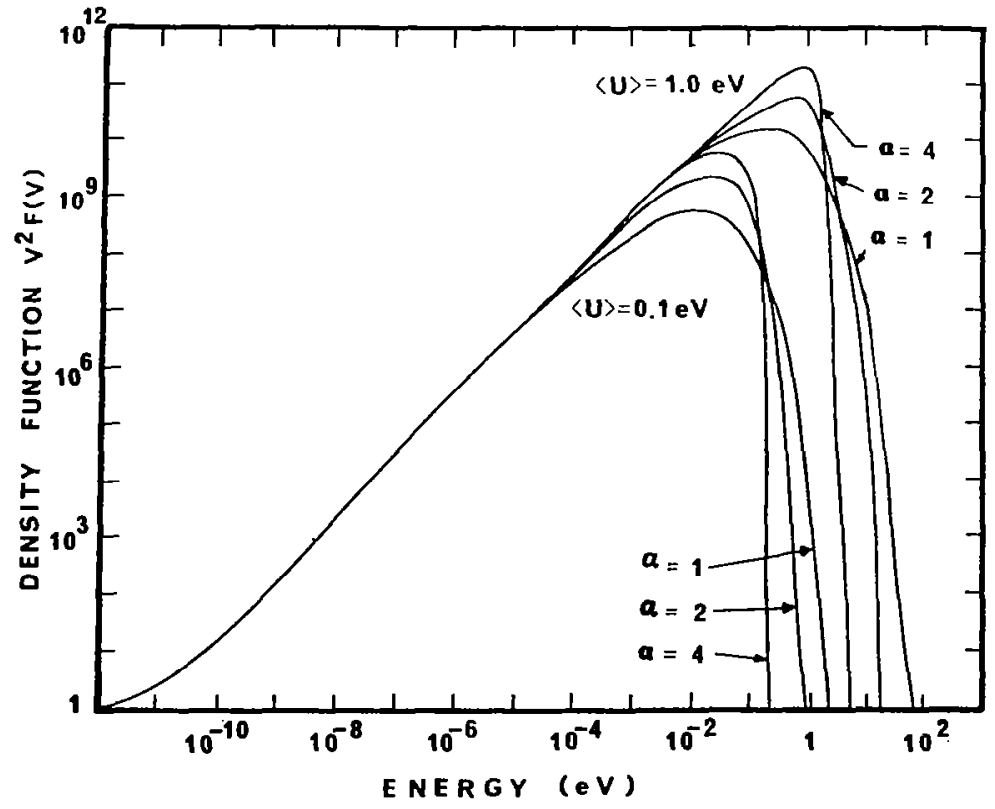

Figure 4. Various calculated forms of the density distribution function $v^{2} f(v)$ versus electron energy. The values of the average electron energy $\langle u\rangle$ is related to the parameter a in the form chosen to represent $f(v) \sim \exp \left(-b v^{a}\right) . \quad a=2$ and 4 correspond respectively to Maxwellian and Druyvestyn distributions. $\mathbf{a}=1$ represents a distribution in which more electrons have energies above the average than is the case for a Maxwelliar distribution. 

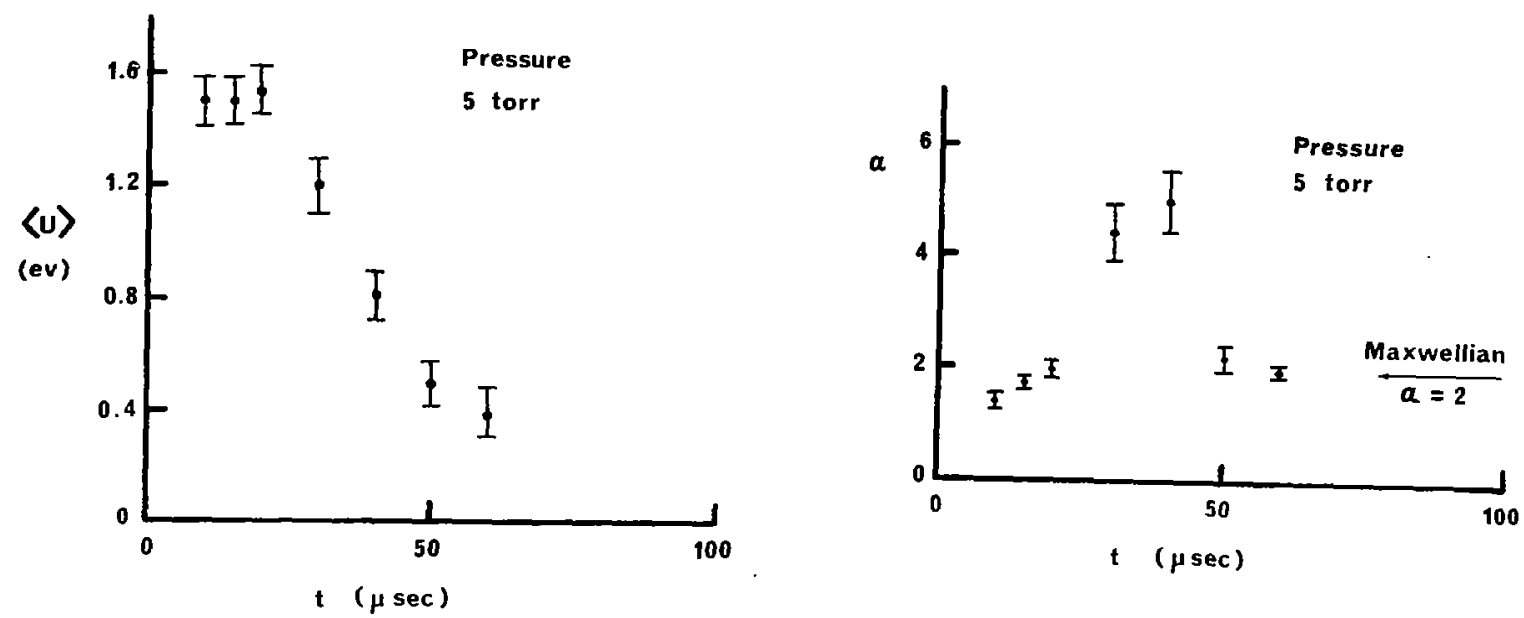

Figure 5. Experimentally determined variation in the mean energy $\langle u\rangle$ and exponential parameter a as function of time in the nitrogen afterglow.

\section{EIECTROMAGNEIIC WAVE PROPAGATION THROUGH MAGNETOPLASMAS - THEORY}

An original theoretical treatment of polarization transformations in terms of a matrix representation based on the concept of orthogonal polarizations has been developed. Part of this was reported in the Proceedings of the IRE (Refs. 18 and 19) and a fuller treatment was given in Technical Report 8 of the Rensselaer Plasma Laboratory (Ref. 20).

Relations were developed for propagation properties of an electromagnetic wave along the magnetic field direction, both for a uniform and an inhomogeneous plasma. Techniques were described for predicting the wave polarization which propagates unchanged through the medium and the wave polarization which propagates with maximum intensity. The expressions derived are briefly summarized below:

The Transformation Matrix

Since only two possible modes corresponding to two possible polarizations can propagate in an anisotropic plasma, then a wave with any other polarization incident on such a plasma will be split into two parts with independent propagation characteristics. Thus the polarization of the incident wave will be continuously transformed as it propagates through the plasma due to the fact that the refractive indices of the two modes are different. We shall assume 
that a transformation matrix $M$ exists which transforms the input polarization $|\pi\rangle$ into the output polarization $\left|\pi^{\prime}\right\rangle$. That is,

$$
\left|\pi^{\prime}>=M\right| \pi>
$$

The assumption of the linear behavior of the medium is generally valid if the propagating wave does not alter the characteristics of the medium. It is therefore a "small signal" theory which could be expected to be valid for communication problems where the energy density of the wave is much less than the energy density of the medium.

We shall first investigate the theoretical form which the transformation matrix $M$ assumes for propagation along the magnetic field in an anisotropic plasma, then present a method for measuring the transformation matrix and discuss the propagation properties which can be determined from such a measurement.

Propagation Along the Magnetic Field

If a wave of arbitrary polarization is propagating along the magnetic field in a uniform anisotropic plasma, how does the polarization of the wave change as the wave propagates?

There are two ways of answering this question depending on whether we describe the polarization in terms of the polarization parameters or the elliptic parameters. We will consider the description in terms of the polarization parameters.

An arbitrary polarization can be represented as

$$
|\pi\rangle=\cos (\theta / 2) \quad\left[\begin{array}{c}
1 \\
\tan (\theta / 2) e^{i \phi}
\end{array}\right]
$$

We can let $|\pi\rangle$ represent an electric field vector of unit intensily. As the wave propagates along the magnetic field the polarization $|\pi\rangle$ will change and the resultant polarization at any point $\left|\pi^{\prime}\right\rangle$ is determined from

$$
\left|\pi^{1}\right\rangle=\mathrm{M}|\pi\rangle
$$

and our objective is to find the form of $M$.

Now the only modes which can propagate along the magnetic field in an anisotropic plasma are the right and left circular waves, corresponding to two of the eigenvectors. Thus, in the two-dimensional principal coordinate system, we can write the polarization of any wave as 


$$
|\tilde{\pi}\rangle=\left[\begin{array}{l}
c_{r} \\
C_{\iota}
\end{array}\right]
$$

where the components $C_{x}$ and $C_{l}$ are given by

$$
C_{r}=(1 / \sqrt{2})[\cos (\theta / 2)+\sin (\theta / 2) \sin \phi-i \sin (\theta / 2) \cos \phi]
$$

and

$$
C_{l}=(1 / \sqrt{2})[\cos (\theta / 2)-\sin (\theta / 2) \sin \phi+i \sin (\theta / 2) \cos \phi]
$$

$\mathrm{C}_{\mathrm{r}}$ propagates with a wave number

$$
\mathrm{k}_{+}=\frac{\omega}{c} \mathrm{n}_{+} \frac{\omega}{c} \sqrt{\mathrm{R}}
$$

while $C_{l}$ propagates with a wave number

$$
k_{-}=\frac{\omega}{c} \quad n_{-}=\frac{\omega}{c} \sqrt{L}
$$

Therefore, at any point along the magnetic field the right and left circular components of the wave will be given by

$$
C_{r}{ }^{\prime}=c_{r} e^{i k}+3
$$

and

$$
C_{l}{ }^{\prime}=c_{6} e^{i k_{-} x_{3}}
$$

We can write these equations in the form

$$
\left[\begin{array}{l}
c_{r}{ }^{\prime} \\
c_{t}
\end{array}\right]=\left[\begin{array}{cc}
e^{i k_{+} x_{3}} & 0 \\
e^{i k x_{3}} & e^{i k_{+} x_{3}}
\end{array}\right]\left[\begin{array}{l}
c_{r} \\
c_{l}
\end{array}\right]
$$

or

$$
\left|\pi^{1}\right\rangle=\widetilde{M}|\tilde{\pi}\rangle
$$


Now the polarizations $|\pi\rangle$ and $|\tilde{\pi}\rangle$ are related by

$$
|\tilde{\pi}\rangle=U|\pi\rangle
$$

and

$$
\left|\tilde{\pi}^{\prime}\right\rangle=U\left|\pi^{\prime}\right\rangle
$$

where $U$ is the two-dimensional unitary transformation given by

$$
U=\frac{1}{\sqrt{2}} \quad\left[\begin{array}{cc}
1 & -2 \\
1 & 2
\end{array}\right]
$$

The inverse of $\mathrm{U}$ is given by

$$
U^{-1}=\frac{1}{\sqrt{2}}\left[\begin{array}{cc}
1 & 1 \\
2 & -2
\end{array}\right]
$$

If we substitute (3) and (4) into (2) we obtain

$$
U\left|\pi^{\prime}\right\rangle=\tilde{M} U|\pi\rangle
$$

Premultiplying by $\mathrm{U}^{-1}$ we obtain

$$
\left|\pi^{\prime}\right\rangle=U^{-1} \tilde{M} U \quad|\pi\rangle
$$

By comparing (7) with (5) we see that

$$
\mathrm{M}=\mathrm{U}^{-I} \tilde{\mathrm{M}} \mathrm{U}
$$

If we now substitute $M$ from (1) together with (5) and (6) we find that

$$
M=\frac{1}{2}\left[\begin{array}{cc}
\left(e^{i k_{+} x_{3}}+e^{i k-x_{3}}\right)-i\left(e^{i k_{+} x_{3}}-e^{i k x_{3}}\right) \\
i\left(e^{i k_{+} x_{3}}-e^{i k x_{3}}\right) & \left(e^{i k_{+} x_{3}}+e^{i k x_{3}}\right)
\end{array}\right]
$$

This is the result which we sought. Its use in (2) will enable us to describe the change in polarization of a propagating wave. 
To generalize the solution of the propegation problem in magnetoplasmas we must take into account the fact that the plasma is probably not uniform and not infinite in extent and that propagation may occur in some arbitrary direction relative to the magnetic field direction. We will show that the information obtained from two polarization measurements after transmission through a magnetoplasma is sufficient to answer the following three questions:

(1) How will the plasma affect the propagation of a wave of any polarization?

(2) What polarization will propagate through the plasma without having its polarization altered?

(3) For what polarization will the wave leaving the plasma have the greatest intensity?

This can be done in terms of orthogonal polarization measurements.

The transformation matrix $M$ characterizes the anisotropic medium. Thus the polarization $|\bar{\pi}\rangle$ which is orthogonal to $|\pi\rangle$ will transform according to

$$
\left|\bar{\pi}^{\prime}\right\rangle=M|\bar{\pi}\rangle
$$

The output polarizations $\left|\pi^{\prime}\right\rangle$ and $\left|\bar{\pi}^{\prime}\right\rangle$ will not, in general, be orthogonal.

If we post-multiply (2) by $|\pi\rangle$ and (8) by $|\bar{\pi}\rangle$, add the resulting equations, and use the relation $|\pi\rangle\langle\pi|+| \bar{\pi}\rangle\langle\bar{\pi}|=1$ we obtain

$$
\left|\pi^{\prime}\right\rangle\left\langle\pi|+| \bar{\pi}^{\prime}\right\rangle\langle\bar{\pi}|=M
$$

which is an expression for the transformation matrix $M$ in terms of the two output polarizations $\left|\pi^{\prime}\right\rangle$ and $\left|\pi^{\prime}\right\rangle$ which result from propagating the two orthogonal polarizations $\langle\pi|$ and $\langle\pi|$ through the anisotropic medium. The transformation matrix $M$ can therefore be determined for any given medium from measurements of the two output polarizations $\left|\pi^{\prime}\right\rangle$ and $\left|\bar{\pi}^{\prime}\right\rangle$, together with measurement of the intensity and phase shift changes of the transmitted signal. Once the transformation matrix has been determined for a given medium one then knows how any other polarization will transform.

The Characteristic Polarizations

From a measurement of the transformation matrix it is possible to determine the polarization of those waves which will propagate through the medium without having their polarization altered. Such a polarization will be called a characteristic polarization and will be designated by $|C\rangle$. This characteristic polarization can be found by the usual method for determining principal axes. For this case the output polarization $\left|C^{\prime}\right\rangle$ is simply some scalar $\lambda$ times the input polarization $|\mathrm{C}\rangle$. That is 


$$
\left|C^{\prime}\right\rangle=M|C\rangle=2|C\rangle
$$

from which

$$
(M-21)|C\rangle=0
$$

which is a set of two homogeneous equations. A non-trivial solution exists only if

$$
\operatorname{Det}(M-2 I)=0
$$

Having measured the transformation matrix $M$ the quadratic equation (1I) can then be solved to give two values for 2 . Using these values of 2 in (10) the characteristic polarizations | C) can then be determined.

The Polarization for Maximum Transmission

The intensity of the input wave will be defined by

$$
I=\langle\pi \mid \pi\rangle
$$

Note that as defined here $I$ is the sum of the squares of the vertical and horizontal components of the wave. If we use the normalized polarizations, then the input wave will be of unit intensity.

The intensity of the output wave is given by

$$
I^{\prime}=\left\langle\pi^{\prime} \mid \pi^{\prime}\right\rangle
$$

We wish to find that input polarization which will resull in the maximum output intensity. Using (2) in (13) we can write the output intensity as

$$
I^{\prime}=\left\langle\pi\left|M^{+} M\right| \pi\right\rangle
$$

where $\mathrm{M}^{+}$is the Hermitian conjugate of $M$. Note from (14) that if $M$ is unitary (i.e. if $M^{+}=M^{-I}$ ) then the output intensity $I^{\prime}$ is equal to the input intensity I given by (I2). Also note that since the complex conjugate of ' $I$ ' is given by

$$
\begin{aligned}
I^{*} & \left.=\left\langle\pi\left|M^{+} M\right| \pi\right\rangle\right\rangle^{*} \\
& =\left\langle\pi\left|M^{+} M\right| \pi\right\rangle=I^{\prime}
\end{aligned}
$$

then $I^{\prime}$ is real. 
We can write the polarization ratio as

$$
Q=\tan (\theta / 2) e^{i \phi}=r+i q
$$

We can write the input polarization as

$$
1 \pi\rangle=\frac{1}{\sqrt{1+r^{2}+q^{2}}}\left[\begin{array}{c}
1 \\
\\
r+i q
\end{array}\right]
$$

Using (15) and the corresponding expression for $|\pi\rangle$ we can write the output intensity from (14) as

$$
I^{\prime}=\frac{1}{1+r^{2}+q^{2}} \quad\left[\begin{array}{ll}
1 & r-i q
\end{array}\right] M^{+} M \quad\left[\begin{array}{c}
1 \\
r+i q
\end{array}\right]
$$

From the measured transformation matrix $M$ we can calculate $I^{\prime}$ from (16). The quantity I' will be a real function of $r$ and $q$. That is,

$$
I^{\prime}=I^{\prime}(r, q)
$$

The maximum value of $I^{\prime}$ will occur when

$$
\frac{\partial I^{\prime}}{\partial r}=0 \text { and } \frac{\partial I^{\prime}}{\partial q}=0
$$

By calculating those values of $r$ and $q$ which satisfy these conditions the polarization which will result in the maximum output intensity can then be determined from (15). The distinction between a maximum and a minimum can be ascertained by either investigating the second derivatives or by using the $M$ matrix to actually calculate the intensity of the output wave.

\section{ELECTROMAGNETIC WAVE PROPAGATION THROUGH MAGNETOPLASMAS-EXPERIMENT}

\section{Free Space Propagation}

Free space propagation at $K$-band frequencies through an argon plasma in a longitudinal magnetic field was used to verify the theory developed in the preceeding section. Construction details of the magnet used were reported in an article in the Review of Scientific Instruments (Ref. 2l). The transmitting and receiving antenna were a pair of conical microwave horns and the polarization of the transmitted signal could be varied by means of the polarizer. This consisted of a length of circular waveguide whose cross-section could be varied from circular to oval and which could be rotated relative to the rectangular input 
waveguide. The polarization emerging was measured using a trimode turnstile junction (Ref. 22).

Polarization measurements at $20 \mathrm{Gc} / \mathrm{s}$ were carried out for an argon plasma at a pressure of 260 microns immersed in a magnetic field of 4500 gauss.

The output polarizations which result from horizontal and vertical input polarizations are shown in Figure 6. From this figure we find that for the initial horizontal polarization

and

$$
|\pi\rangle=\left[\begin{array}{l}
1 \\
0
\end{array}\right]
$$

$$
\left|\pi^{\prime}\right\rangle=\left[\begin{array}{ll}
1.06 & \\
0.31 & \angle-27^{\circ}
\end{array}\right]
$$

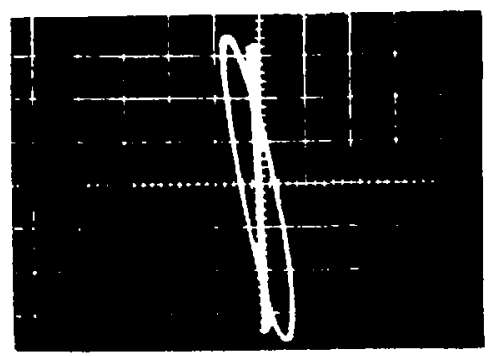

a

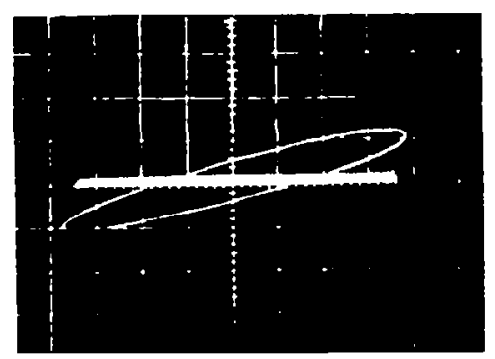

b

Figure 6 Transformation of oxthogonal polarizations. Case (a) initial polarization is horizontal. Case (b) initial polarization is vertical. 
while for the initial vertical polarization

$$
|\pi\rangle=\left[\begin{array}{l}
0 \\
1
\end{array}\right]
$$

and

$$
\left|\pi^{\prime}\right\rangle=\left[\begin{array}{ll}
0.23 & \angle 150^{\circ} \\
1.03 &
\end{array}\right]
$$

It is important to note that the same relative phase relation exists between the $E_{1}$ component of $|\pi\rangle$ and the $E_{1}$ component of $\left|\pi^{\prime}\right\rangle$ as between the $E_{2}^{1}$ compunent of $|\pi\rangle$ and the $E_{2}$ component of $\left|\pi^{\prime}\right\rangle$ This fact cannot be obtained directly from Figure 6 , but can be determined independently by comparing the phases of the signals representing the $E_{1}$ component of $\left|\pi^{\prime}\right\rangle$ and the $E_{2}$ component of $\left|\bar{\pi}^{\prime}\right\rangle$ with the phase of a reference signal.

Using these results in (9) we can readily calculate the $M$ matrix to be

$$
M=\left[\begin{array}{ccc}
1.06 & 0.23 & \angle 150 \\
0.31 & \angle-27^{0} & 1.03
\end{array}\right]
$$

The characteristic polarizations can now be determined. The two eigenvalues are

$$
\lambda^{(1)}=0.92-i 0.23
$$

and

$$
\lambda^{(2)}=1.17+i 0.23
$$

The characteristic polarization corresponding to $\lambda^{(1)}$ is given by

$$
\mathrm{E}_{2} / \mathrm{E}_{1}=1.20 \angle 89^{\circ}=\tan (\theta / 2) \mathrm{e}^{i \phi}
$$

which corresponds to a right-handed wave whose orientation angle is $86^{\circ}$. and whose axial ratio is .84 .

The result of propagating this polarization through the plasma is shown in Figure 7a. The two polarizations are superimposed on each other indicating that the initial polarization was unaltered. 


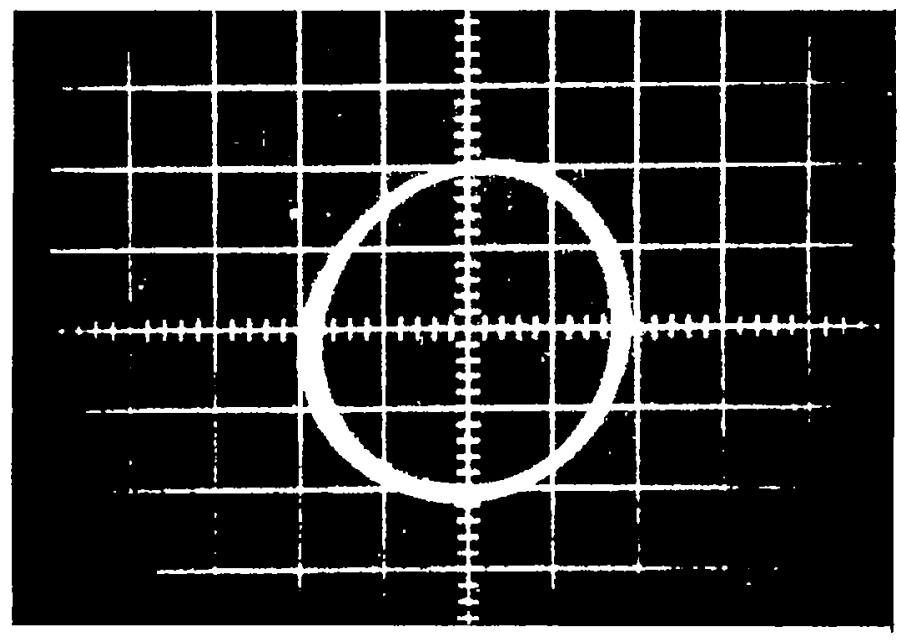

a

Figure $7(a)$ Transformation of the characteristic polarization. Input and output ellipses coincide.

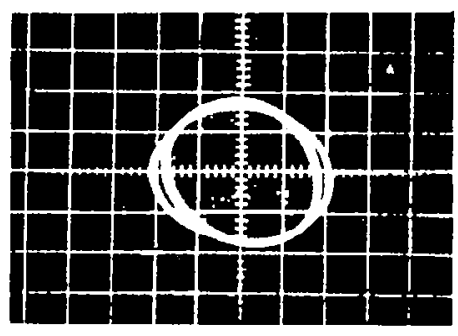

b

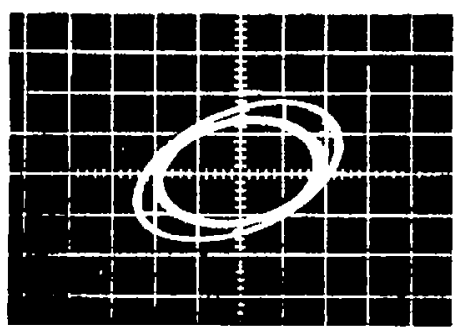

C

Figure $7(b)$ Transmission of the appropriate input polarization (inner ellipse) which yields minimum output signal intensity (outer ellipse).

Figure 7(c) Transmission of the appropriate input polarization (inner ellipse) which yields maximum output signal intensity (outer ellipse). 
The polarization which results in the maximum transmission can also be determined. Using (17) and (16) we can write the output intensity as

$$
I^{\prime}=\frac{1.22+1.11 r^{2}+1.11 q^{2}+0.14 r-.54 q}{1+r^{2}+q^{2}}
$$

The condition $\partial I^{\prime} / \partial r=0$ yields

$$
q^{2}-r^{2}+7.7 r q-1.57 r+1=0
$$

while the condition $\partial I^{\prime} / \partial q=0$ yields

$$
q^{2}-r^{2}-0.52 r q-.41 q-i=0
$$

These equations can be solved simultaneously for $r$ and $q$. The two solutions are

$$
r=0.16, \quad q=-0.82
$$

and

$$
r=-0.27, \quad q=1.19
$$

From (15) we find that these two solutions correspond to the polarizations

$$
|\pi\rangle_{\min }=\left[\begin{array}{ll}
0.77 & \\
0.84 & \angle-79^{\circ}
\end{array}\right]
$$

and

$$
|\pi\rangle_{\max }=\left[\begin{array}{ll}
0.87 & \\
0.83 & \angle-75^{\circ}
\end{array}\right]
$$

respectively. The polarization given by (18) results in a minimum intensity while (19) results in a maximum intensity as can be seen by applying the $M$ matrix (17) to these cases.

The results of propagating waves with the polarizations (18) and (19) through the plasma are shown in Figure $7 \mathrm{~b}$ and $7 \mathrm{c}$. 


\section{Guided Wave Propagation}

An $X$-band all metal waveguide cell was developed which incorporates modified turnstile junctions, allowing measurement of the change of polarization of microwaves propagating through a magnetoplasma. The work was reported in an article in the Review of Scientific Instruments (Ref. 3).

This metal waveguide cell has advantages over the type of discharge vessel used by other workers for propagation studies in longitudinal magnetic fields. Glass discharge vessels enclosed in metal waveguide have several disadvantages: construction and assembly are more difficult than with an all-metal system, and uncertainties in the appropriate boundary conditions are introduced by the presence of the glass. Where a longitudinal magnetic field is present, it is also advantageous to have both discharge electrodes parallel to the axis of the vessel and to the direction of microwave propagation. This axial electrode symmetry minimizes wall effects. It has been found experimentally that with asymmetrical electrode construction, similar to that used by other workers, the plasma may be displaced in the magnetic field to the extent of striking one of the walls of the glass vessel.

The signals from the four waveguide output ports (see Fig. 8) provide information about the change in polarization of the propagated EM wave due to the magnetoplasma when the discharge cell is operated in a longitudinal magnetic field. The response of crystals mounted on a pair of orthogonal ports at the output end can be used to obtain the magnitude of the horizontal and vertical components. The outputs of the other two ports are combined at a hybrid junction with the difference in electrical length between the two ports and the hybrid junction adjusted to a multiple of $\pi / 2$. The magnitudes of the signals from crystals mounted on the $H$ and $E$ arms of the hybrid junction give the magnitude of the right and left circular components of the wave.

If the amplitudes of the circular wave components entering and leaving th magnetoplasma are $\mathrm{C}_{+}$and $\mathrm{C}_{-}$and $\mathrm{C}_{+}{ }^{\prime}$ and $\mathrm{C}_{-}{ }^{\prime}$ respectively, then

$$
C_{+}{ }^{\prime} / C_{-}^{\prime}=e^{-\left(k_{+}-k_{-}\right) L} C_{+} / C_{-}
$$

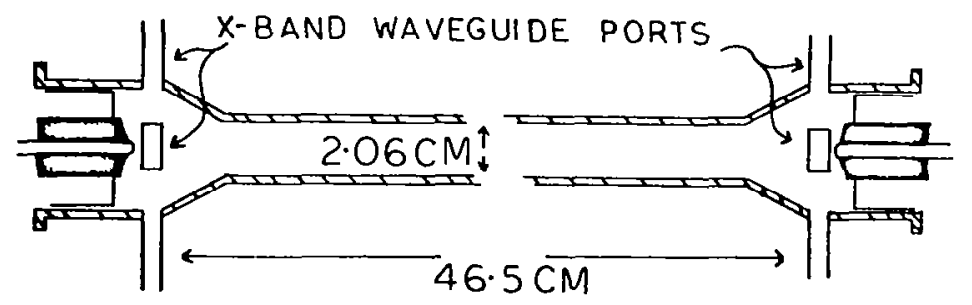

Figure 8. All metal $\mathrm{X}$-band microwave discharge cell. The electrodes form part of the coaxial matching stub assembly of the modified turnstile junction at each end of the cell. 
where $k_{+}=$the propagation constant of the appropriate circular component

$-\beta_{ \pm}+i \alpha_{ \pm}$

$\beta_{ \pm}=$the phase shift per unit length

$\alpha_{ \pm}=$the attenuation per unit length

$\mathrm{L}=$ the length of the magnetoplasma in the direction of propagation.

It is possible to evaluate the phase shift and attenuation parameters separately.

The rotation of the plane of polarization per unit length is given by

$$
\psi=\left(\beta_{+}-\beta_{-}\right) / 2
$$

When the electron collision frequency $v$ is very much less than the microwave angular frequency $\omega, \psi$ can be used to determine the average electron density $\mathrm{n}_{e}$ directly. It is necessary to know the radius of the region contuining the plasma relative to the radius of the guide, so that visual inspection of the plasma column in the magnetic field is required.

When $\omega$ is close to the cyclotron frequency, $\psi$ will be dependent on both $\mathrm{n}_{\mathrm{e}}$ and $\nu$. The collision frequency $\nu$ may be calculated from the attenuation factor.

Microwave Lens System

A microwave lens system operating at $K$ band frequencics was developed for plasma diagnostics. This work was reported in a INASA report (Ref. 23). Two types of lens were considered, the dielectric lens and the Fresnel half-period zone plate (Ref. 24). The superiority of the Fresnel plate was demonstrated in better control of bandwidth and gain and in concentrating most of the microwave energy close to the axis through the center of the lens.

PLASMA STABILITY AND TRANSPORT IN MAGNETIC FIELDS

Analysis of the Modes of the Helical Instability of the Helium Positive Column

Experimental investigations have been carried out on factors affecting the stability and containment of a plasma in a longitudinal magnetic field. The work has been done on positive column discharges in helium at pressures of 1 torr in which, above a certain critical value of the magnetic field $\mathrm{B}_{\mathrm{C}}$, a helical instability develops. A mode analysis of the instabilities generated has been made and the effects of a small transverse magnetic field studied in detail. This work has been reported in an AIAA paper (Ref. 25) and in Physical Review Letters (Ref. 26). 
Two sets of four radial probes, each set being in a plane perpendicular to the current flow, were used. The $\mathrm{m}=0,1$, and 2 modes were identified by appropriate phase shift measurements.

The $\mathrm{m}=0$ oscillation vanished when the applied electric and magnetic fields were precisely aligned but could be generated if a transverse field component as small as 0.5 gauss was present in a total magnetic field of around 2 kilogauss. The amplitude of the $\mathrm{m}=0$ and $\mathrm{m}=1$ mode oscillations, shown in Figure 9 as a function of the transverse magnetic field $B_{T}$, demonstrate clearly the effect of $B_{T}$

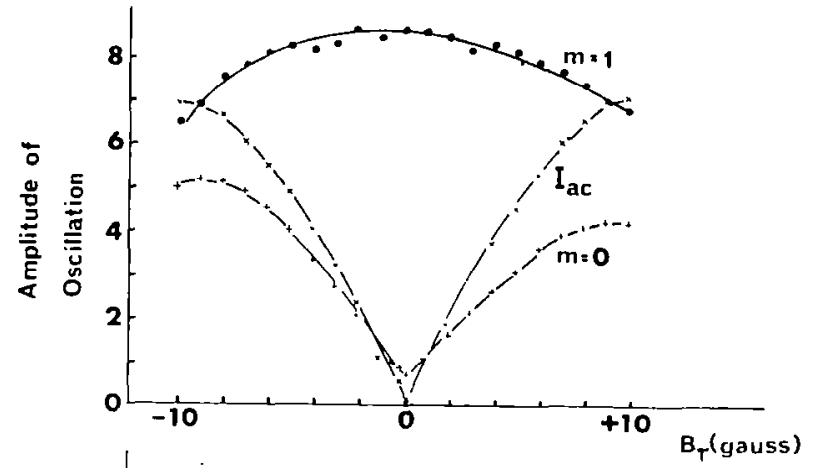

Figure 9. Upper: amplitudes of the oscillations in discharge current $\left(I_{a c}\right)$ and the $m=0$ and $m=I$ modes as functions of the magnetic field. Lower: coupling coefficients, $\mathrm{C}_{0}=|\mathrm{m}=0| / \mathrm{m}=1 \mid$ and $C_{i}=I_{\text {ac }} /|\mathrm{m}=1|$, as functions of the transverse magnetic field.

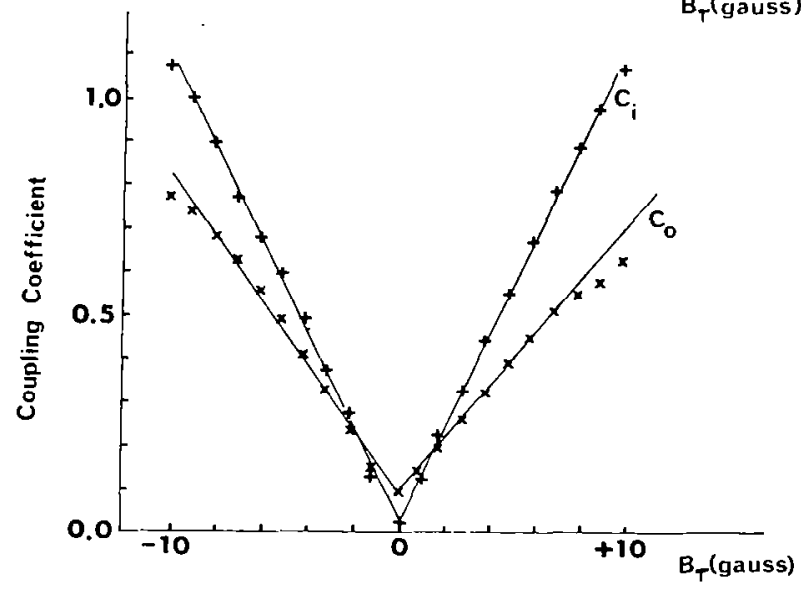

on the $\mathrm{m}=0$ mode. The amplitude of the two oscillations is similar provided $B_{T}$ is of order 10 gauss. It is also found that in the region outside the longitudinal magnetic field the amplitude of the $m=1$ oscillation dies away whereas that of the $m=0$ oscillation remains almost constant.

The observed $\mathrm{m}=0$ oscillation is interpreted not as an unstable mode of the helix as in the $m=1$ oscillation but rather as a nonlinear effect arising from the interaction of the helical mode with the asymmetry introduced by the transverse magnetic field.

'The effect of $\mathrm{B}_{\mathrm{T}}$ on the stability of the $\mathrm{m}=1$ and $m=2$ modes was analysed mathematically and a modified form of the Kadomtsev criterion obtained which predicted mode switching for a given longitudinal field as $B_{T}$ was increased. The experimental results shown in Figure 10 verify this analysis. The effect of small transverse magnetic fields on the overall stability considerations of the plasma has thus been shown to be quite significant. 


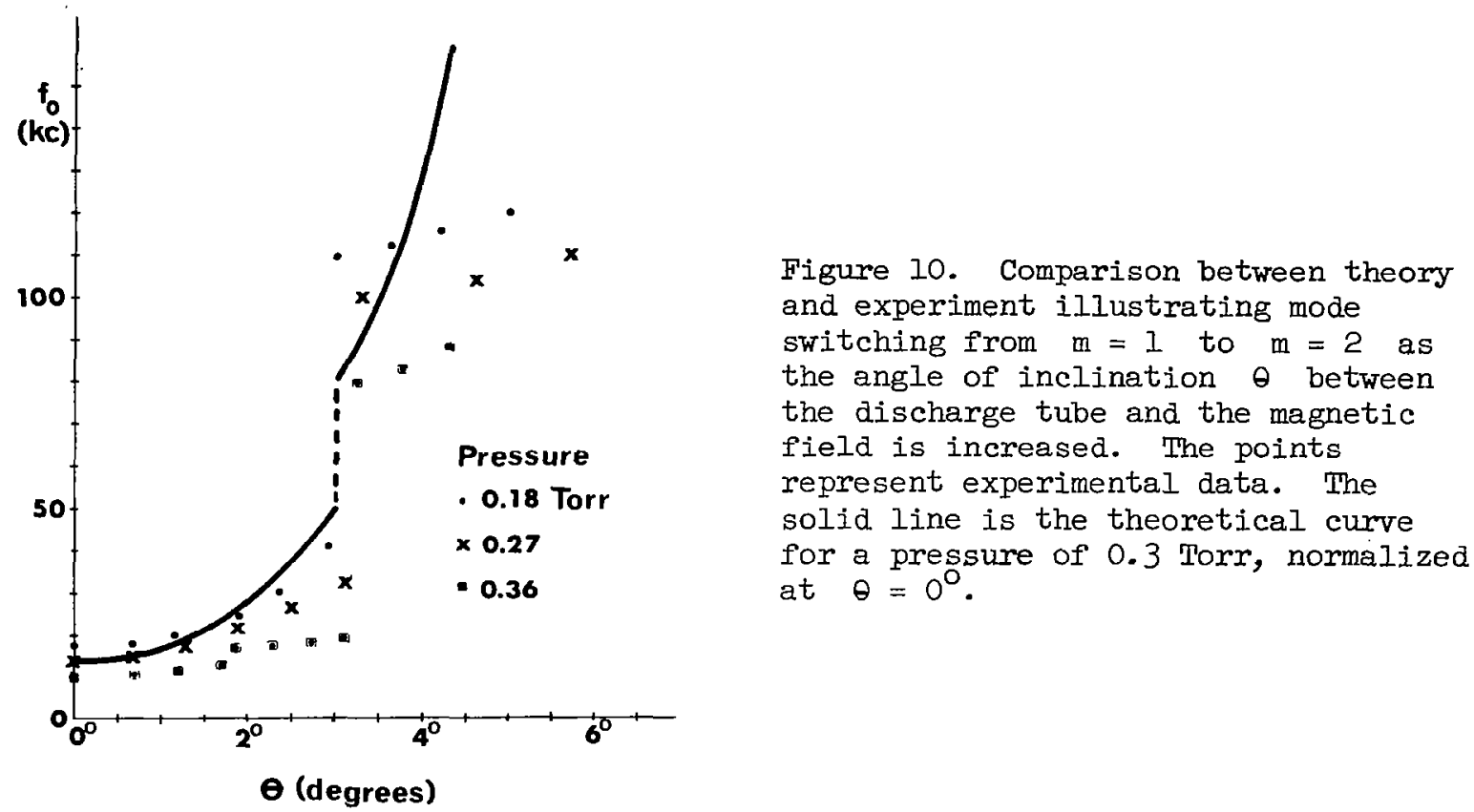

Subthreshold Excitation

It is common practice to use a linearized theory to predict the critical magnetic field $B_{\text {and }}$ the characteristic of the fully developed helical instability, even though nonlinear effects must be involved in the anomalous state of the positive column which is studied experimentally. A subthreshold experiment was carried out by exciting a pair of probes in the discharge and measuring the received signal at another pair of probes $40 \mathrm{~cm}$ farther down the column as the magnetic field was increased to a value just below $B_{c}$.

A typical result is shown in Figure 11. A well-developed resonance in the received signal occurs as $B$ nears $B_{C}$. The sub-threshold resonance is within $1 \%$ of that of the self-sustaining osciliations which the plasma exhibits at $B=B_{C}$. This experimental check serves as a test of the linear assumption in the Kadomtsev theory (Ref. 27). This work has been reported in an Applied Physics Letter (Ref. 28).

The sub-threshold technique was also applied to a study of the separate $m=1$ and $m=2$ modes of the Kadomtsev instability. The theoretical formulation derived previously indicates that when the transverse field component $B_{T}$ exceeds a certain value the $m=2$ mode becomes less stable than the $m=1$ mode. Experimentally obtained sub-threshold response spectra confirming the effect are shown in Figure 12, indicating that the $\mathrm{m}=2$ mode is a genuinely independent mode of instability. 
Received

Signal
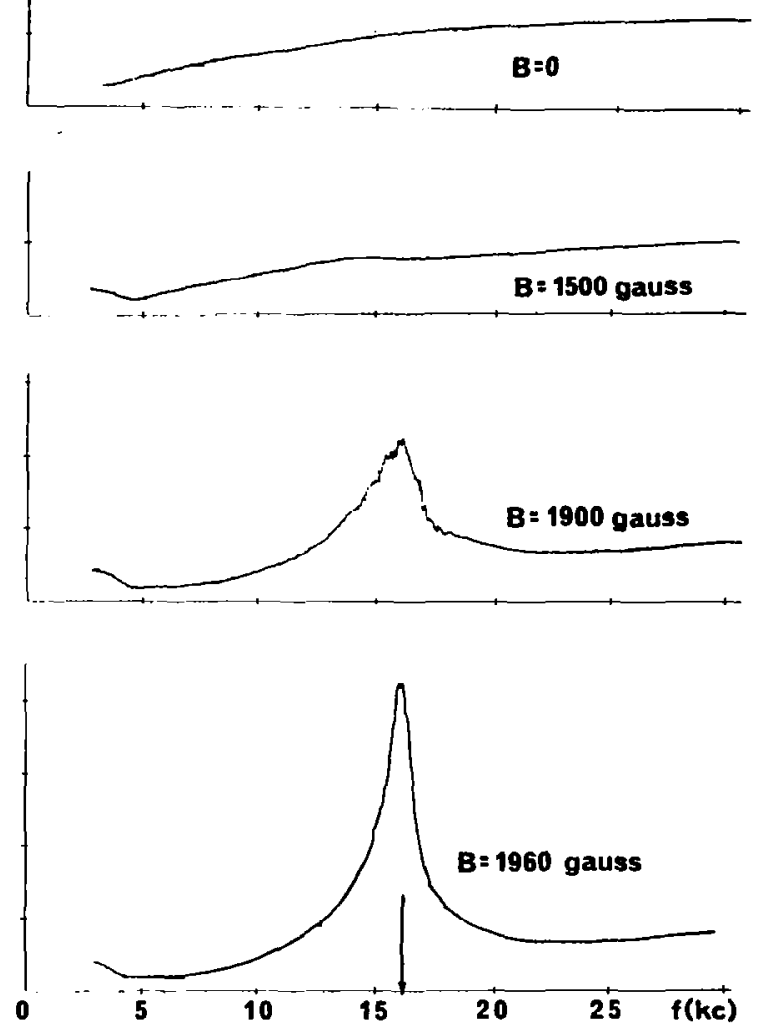

Received

Signal
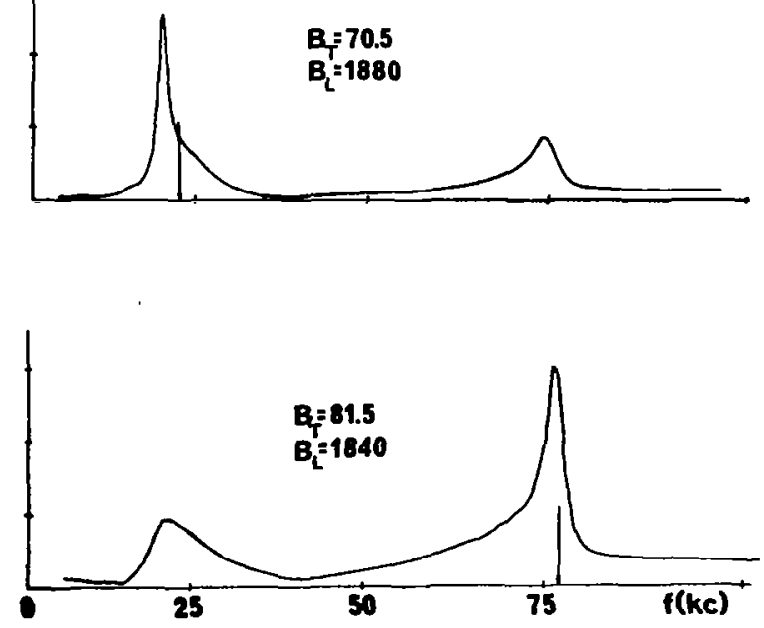

Figure 11. Signal received at second probe set as a function of the frequency of the source connected across the first probe set. At the critical field strength (2l00 gauss), the plasma exhibited self sustaining oscillations at $16.3 \mathrm{Kc} / \mathrm{s}$.

Figure 12. Sub-threshold response spectra as a function of the applied transverse magnetic field. The arrows indicate the frequency of self-sustaining oscillations that appear when the magnetic field is increased above the critical value $\left(B_{c}\right)$. 
Preliminary experiments aimed at stabilizing the plasma by means of quadrupole magnetic fields (an Ioffe bar configuration) have shown a marked effect as indicated by rigure 13. However this geometry is difficult to analyse mathematically.

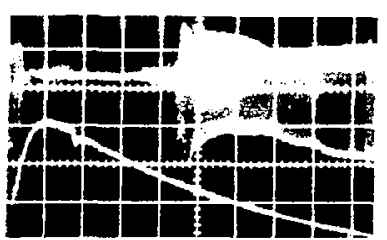

$$
\begin{aligned}
& V_{\text {probe }}: 2 \mathrm{v} / \mathrm{cm} \\
& I_{\text {stab. }}: 40 \mathrm{~A} / \mathrm{cm}
\end{aligned}
$$

Figure 13. Stabilization of the discharge by a quadrupole magnetic field. Top trace: AC voltage picked up by a probe. Bottom trace: current through the quadrupole windings.

$5 \mathrm{~ms} / \mathrm{cm}$

A simpler magnetic field geometry which should, in principle, yield significant results in terms of the development of the helical instability is that of an azimuthal B field. Work is in progress on setting up such a system using a central current-carrying conductor along the axis of a hollow annular discharge cell.

\section{Cesium Plasma Cell}

A special cesium plasma cell has been constructed which has several novel design features. There are three distinct regions each characterized by separate vacuum systems: two electron gun assemblies and a neutral plasma region. The plasma source consists of a cesium reservoir with 1000 hollow stainless steel rods which inject the neutral cesium atoms directly on to the hot tungsten collector. When run double-ended, that is, with cesium sources at both ends of the cell, in a magnetic field of prder several kilogauss, it is expected to achieve densities of the order of $10^{14} \mathrm{~cm}^{-3}$.

The Initial Value Problem of Plasma Transport Phenomena

A theoretical study has been made of the initial value problem involved in the mathematical treatment of rapidly varying plasma phenomena. The transport of electrons from a given initial state in a neutral-collision-dominated plasma is treated for the cases of diffusion, flow in a uniform electric field, and flow across a magnetic field. This work has been reported in a NASA Contractor report (Ref'.29). 
The adequacy of the classical theory of diffusion derived from Fick's law, (particle flow proportional to density gradient), has been questioned when it is applied to plasma phenomena which vary rapidly with time. The macroscopic telegrapher's equation includes the effect of particle inertia but does not necessarily provide a more accurate representation of the particle flow than Fick's law. It does however show that flow of fluid from a region which is initially at a higher density to other regions involves a particle flow which decays exponentially in time, and that the flow has a leading edge that moves with the thermal velocity. There is no prediction of an infinite velocity of propagation as in the formulation derived from Fick's equation.

As illustrated in Figure 14 the telegrapher's equation describes a flux of particles moving at the thermal velocity which is not predicted by the classical equation. While this appears to be a refinement in the description it also serves to point up the defect of all macroscopic descriptions because the leading edge, which apparently is so sharp, is in fact drawn out in space in a way which depends upon the number of particles in each velocity class in the initial distribution. To recover an accurate description of the particle flux we must therefore resort to a microscopic formulation of the problem with the velocity distribution function explicit and with particular attention paid to the initial conditions. We use the Boltzmann equation to discuss the problem of time-dependent diffusion from given initial conditions in a Lorentz gas in the presence of external fields and spatial gradients.
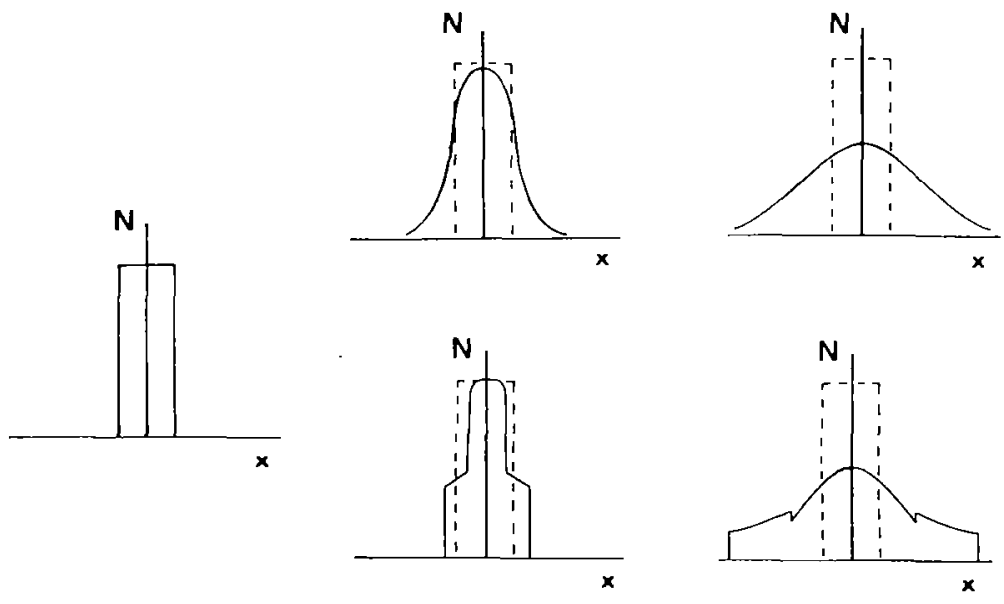

Figure 14. Spatial diffusion of particles from an initial rectangular diffusion in configuration space. Comparison between the Fickian (upper) and telegrapher's equation (lower) descriptions is shown. 
The Boltzmann equation for the electrons can be written in the form:

$$
\frac{\partial f}{\partial t}+v \cdot \frac{\partial f}{\partial r}+R \cdot \frac{\partial f}{\partial v}=\int d v_{N} \int d \omega s(x) g\left(f^{\prime} F^{\prime}-f F\right)
$$

where $R$ is the external force per unit mass, $F(r, v)$ the number density of neutral particles on the space of $(r, v)$ which is assumed to be constant in time, $v_{N}$ the neutral particle velocity, $g$ the relative speed between the electrons and neutral particles, $s(x)$ the scattering cross section in terms of the scattering angle $\chi$ and $d \omega=\sin x d x d \phi$ is the element of volume in scattering space. The primed functions are evaluated at velocities that are prc-collision with respect to $v$ and $v_{N}$ which are then regarded as post-collision velocities. Since we ignore the recoil of the massive neutral particle, $F$ is equal to $F^{\prime}, g=V$ and $V^{\prime}$ depends only on $\chi$. Then the $v_{N}$ integration can be replaced by the density $\mathbb{N}$ of neutral particles in physical space.

For simplicity we restrict the treatment to the case where $\mathrm{R}$ and $\mathrm{N}$ do not vary in the spatial $y$ and $z$ directions. Then if $f$ satisfies the Boltzmann equation, so do the $y$ and $z$ derivatives. We take these derivatives to be zero every where initially, and it follows that they remain zero.

The integro-differential equation may be expressed as a set of differential equations by using spherical coordinates $(v, \theta, \phi)$ in velocity space and expanding $f$ in spherical harmonics

$$
f(x, v, t)=\sum_{\ell=0}^{\infty} \sum_{m=-\ell}^{\ell} f_{\ell m}(x, v, t) \mathrm{Y}_{\ell \mathrm{m}}(\theta, \phi) .
$$

A hierarchy of equations is then obtained in the $f \mathbf{l}$. For the description of deviations from isotropy that develop in a limited time it is appropriate to focus attention on $f_{0 O}$ (denoted by $f_{0}$ ) and the $f_{I m}$, neglecting terms of higher order in $\boldsymbol{\ell}$. Introducing the real functions

$$
f_{1}=\frac{f_{11}+f_{1,-1}}{\sqrt{2}}, \quad f_{2}=\frac{f_{11}-f_{1,-1}}{\sqrt{2} i} \text { and } f_{3}=f_{10}
$$

we obtain a truncated hierarchy of equations.

In the absence of a magnetic field, it is convenient to use the Boltzmann equation in Cartesian velocity coordinates, $\bar{v}=\left(v_{1}, v_{2}, v_{3}\right)$. Then $f$ is $f_{0}+v^{-1}(\bar{v} \cdot \vec{f})$ where $\bar{f}$ is $\left(f_{1 l}, f_{2}, f_{3}\right)$. If $f$ is initially 1 sotropic then we always have $f-g_{0}+g_{1}$ where $g_{0}=f_{0}$ is the part of $f$ that is even with respect to $v_{1}$ and $g_{1}=v^{-1}\left(v_{1} f_{1}\right)$ is the part of $f$ which is odd.

The truncated hierarchy then becomes

$$
\frac{\partial g_{0}}{\partial t}+v \frac{\partial g_{1}}{\partial x}-\varepsilon \frac{\partial g_{1}}{\partial v_{1}}=0
$$


and $\frac{\partial g_{1}}{\partial t}+v \frac{\partial g_{0}}{\partial x}-\varepsilon \frac{\partial g_{0}}{\partial v_{1}}=-v g_{0}$

where. $\varepsilon=\frac{-e}{m} E=R$

\section{Diffusion}

The application of this approach to free diffusion will be briefly indicated in this report. Further details are given in reference 30 where the case involving electric fields is discussed.

In the case of diffusion $\boldsymbol{\varepsilon}=0$ and the two equations in the hierarchy may be combined to give

$$
\frac{\partial^{2} g_{i}}{\partial t^{2}}+v \frac{\partial g_{i}}{\partial t}=v_{i}^{2} \frac{\partial^{2} g_{i}}{\partial x^{2}}
$$

for both $g_{Q}$ and $g_{l}$ and hence for $f$ itself in the present approximation. It is interesting to note that this is a telegrapher's type equation at the microscopic level.

For an electron fluid composed of $\mathrm{n}_{0}$ electrons initially concentrated in a delta function so far as the x-variations are concerned, the initial density and its time derivative are

$$
\mathrm{n}(\mathrm{x}, 0)=\mathrm{n}_{0}, \delta(\mathrm{x}) \text { and } \mathrm{n}_{\mathrm{t}}(\mathrm{x}, 0)=0
$$

where $n_{t}$ is the time derivative of the density. Further, we use the initial condition of an isotropic distribution in velocity space

$$
g_{0}=F(x, v) \text { and } g_{I}=0 \text {, }
$$

which imply that

$$
f(x, v, 0)=F \text { and }\left(\frac{\partial f}{\partial t}\right)_{t=0}=-v_{1} \frac{\partial F}{\partial x}
$$

Mlso $F(x, v)=n_{0} \quad \delta(x) \quad G(v)$

where $G(v)$ is the initial velocity distribution. 
It follows that $f=0$ for $|x|>\left|v_{1}\right| t$ and elsewhere $f$ is given by

$f=\exp \left(-\frac{v t}{2}\right) G(v) n_{0} \delta(x-v t)+\nu \frac{\exp (-v t / 2)}{2\left|v_{1}\right|}, n_{0} G(v)\left[I_{0}-\left(\frac{v_{1} t+x}{v_{1} t-x}\right)^{I / 2} I_{1}\right]$ where the Bessel functions are evaluated at $\left[\nu /\left(2\left|v_{1}\right|\right)\right]\left[v_{1}^{2} t^{2}-x^{2}\right]^{1 / 2}$. We
use the modified Bessel functions which are related to the ordinary Bessel functions by $I_{n}(x)=i^{n} J_{n}(i x)$.

This equation describes a fluid composed of electrons of a particular velocity class which shoots out a leading edge followed by a residue that becomes Fickian after long times. Thus the behavior is similar to that described by the macroscopic telegrapher's equation. On the other hand the macroscopic density derived from the two approaches can be substantially different. For example, if the initial distribution of velocities is Maxwellian, then the density of particles associated with the free-streaming is such that there is no leading edge. This is illustrated in Figure 15 which points up the differences between the two macroscopic approaches and the microscopic approach in a qualitative way. The separation of collided and uncollided particles is made explicit.

It is important to notice that the description based on the macroscopic telegrapher's equation is least accurate at early times where it differs most from the classical description. In fact for a given injtial condition there may be no time at which the macroscopic telegrapher's equation adequately represents the correction to the classical diffusion equation. It is not possible therefore to identify any version of the macroscopic theory as the preferred version during the early stages of diffusion where the descriptions differ. The flow depends strongly on the initial distribution of velocities, which is hidden in the macroscopic descriptions. Moreover the constraints used to terminate the hierarchy of macroscopic equations, which must be arbitrary to a certain extent, can have a significant effect upon the result, particularly for short times. In order to derive an appropriate description we conclude that a microscopic analysis must be followed.

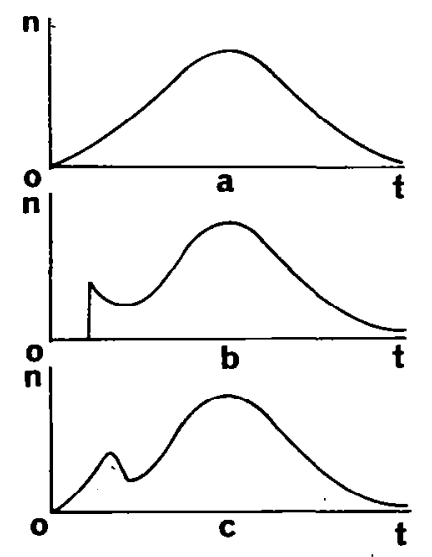

Figure 15. Temporal diffusion of particles past a point $x$ for a gas initially localized at $\mathrm{x}=0$. Comparison between (a) the classical or Fickian description, (b) the macroscopic description based on the telegrapher's equation and (c) the microscopic description based on the Boltzmann equation with a Maxwellian distribution of initial velocities. 


\section{ATTEMPT TO GENERATE PLASMA OSCILTATIONS WITH A RELATIVISTIC EIECTRON BEAM}

It has been known for many years that plasma electron oscillations occurringin gas discharge vessels can be detected with probes. More recently, i.t has been shown that these oscillations can cause radiation that can be detected by a sensitive receiver placed outside the discharge vessel (Refs. 30,31). Looney and Brown (Ref. 32) showed that, at least in their specially constructed vessel, the frequency and amplitude of plasma oscillations depend strongly upon the state of electrode sheaths. However, any modulated beam, passing through a plasma, may set up plasma oscillations, providing one of the Fouricr components of the beam is an rf current whose frequency is close to the plasma frequency. Such modulated beams would be expected to exist in discharges because of the beam-plasma instability (Refs. 33, 34,35).

An experiment has been performed in which a bunched beam from the Rensselaer Linear Accelerator was fired through a decaying plasma. A sensitive receiver was placed outside the vessel to monitor the level of microwave radiation resulting from the beam-plasma interaction.

This experiment possessed the following features:

1. The use of a decaying plasma eliminated the possibility that external power circuits were furnishing the power to maintain oscillations. All the power for oscillations had to come from the beam.

2. Since the plasma was allowed to decay, it approximated a cold plasma.

3. By the very nature of the accelerator, the beam was pre-modulated, so that sheath modulation or instability modulation by the plasma was not required. Conversely, the beam electrons moved with almost the speed of light, so that any oscillating fields in the plasma could not impress additional modulation of the beam density. Furthermore, the beam was not injected through an energized electrode, as in the Looney-Brown experiment, or other experiments using a separate electron gun.

4. The beam could be injected. into the plasma at any pre-selected time during the plasma decay in 0.1 microsec. bursts. During this time the plasma density decayed by about $1 / 100$ of $1 \%$. Also, because the high energy electrons have a very slight tendency to ionize the argon gas, the beam did not reverse the decay of the plasma density.

5. Probes were not inserted into the beam-plasma interaction region, since it was feared that these might perturb the plasma or account for spurious detection of oscillations.

The sixteenth harnonic of the beam's fundamental frequency is $20.800 \mathrm{kMc}$. The Linac beam was fired through the decaying plasma when the plasma frequency near the center of the vessel was close to this frequency. - If the bunched beam from the accelerator is regarded as the Fourier series superposition of a de current and rf currents, then the amplitude of the sixteenth harmonic current 
is about $1 / 3$ of the amplitude of the fundamental current. It was expected that the current's sixteenth harmonic would interact strongly with the plasma, while the higher and lower harmonics would interact weakly. Then a peak beam current of $0.3 \mathrm{amp}$ would have the same effect as a 0.1 amp rf current.

The discharge vessel was $28 \mathrm{~cm}$ long and $5 \mathrm{~cm}$ in diameter. Metal caps at the ends of the vessel acted as electrodes and also as entrance and exit windows for the accelerator beam. The vessel was filled with argon gas to 0.115 torr and a discharge was initiated by a $60 \mathrm{microsec}, 15$ amp pulse from a plasma pulser. A microwave bridge was used to monitor the plasma density in the vesscl. The interferometric fringe pattern, exhibited on an oscilloscope, displayed no jitter, indicating that the pulser created essentially the same density each time.

The accelerator beam was fired along the axis of the vessel. The region of interaction between the beam and the plasma was then a cylindrical region coaxial with the vessel. Its length was the length of the vessel, $28 \mathrm{~cm}$, and its diameter was the diameter of the beam, $1.6 \mathrm{~cm}$ (Ref. 36).

Due to the diffusion of plasma electrons and ions to the walls of the vessel during the decay, the plasma electron density in the interaction region was nonuniform. The plasma density at the boundary of the interaction region was estimated to be $10 \%$ lower than the axial density, making the plasma frequency there 5\% lower. This means that in the interaction region the beam passed through a distribution of plasma frequencies.

Ten times each second the plasma was pulsed and allowed to decay. Then, at a pre-determined time during the decay, the Linac beam was fired for 0.1 microsec. The time delay between pulsing the plasma and firing the Iinac beam could be varied in 2.5 microsec steps. The longer the time delay between pulsing the plasma and firing the Linac beam, the smaller the plasma frequency at any point when the beam passes through it. It was determined, by examining the interferometric pattern, that in 2.5 microsec the plasma frequency near the center of the vessel changed several tens of Mc. Covering the entire decay period in such small steps assured that the beam and the plasma would pass close to resonance. This was augmented by the fact that the interaction region contains a distribution of plasma frequencies.

A $K$-band microwave horn was placed outside the vessel and was connected to a superheterohyne receiver. The receiver bandwidth was $8 \mathrm{Mc}$ and its sensitivity was $2.5 \times 10^{-11}$ watts. The accelerator fundamental frequency and the receiver frequency were measured with wavemeters consisting of resonant cavities. The receiver frequency was varied in $5 \mathrm{Mc}$ steps in the range $20,690 \mathrm{Mc}$ to $20,890 \mathrm{Mc}$.

The receiving antenna had an effective area of $75 \mathrm{~cm}^{2}$. It was located $25 \mathrm{~cm}$ from the vessel, midway between the ends of the vessel, where it intercepted $1 / 50$ of the radiation at right angles to the axis of the vessel. The receiver was expected to respond to radiation at a higher level than $2.5 \times 10^{-11}$ watts. Using a peak current of 0.3 amp (equivalent to an rf current of 0.1 amp) it was not possible to stimulate oscillations at a sufficiently high level to excite the receiver. It can therefore be stated that the power emitted from the vessel was less than $1.25 \times 10^{-9}$ watts, or $0.4 \times 10^{-10^{0}}$ watts per $\mathrm{cm}$ of path transversed by the beam. A detailed report has been prepared (Ref. 37). 


\section{REF'ERENCES}

1. Taft, W. C., K. C. Stotz and E. H. Holt, "A Gated Radiometer for Pïasma Afterglow Study", IFEF'Trans. IM-12, 90-96 (1963).

2. Holt, E. H. and K. C. Stotz, "Plasma Waveguide Cell for Afterglow Measurements", Rev. Sci. Instr., 34, 1285 (1963).

3. Noon, J. I., J. F. Reynolds and E. H. Holt, "X-Band Waveguide Cell for Study of Microwave Propagation through Magnetoplasmas", Rev. Sci. Instr., 36, 622-625 (1965).

4. Stotz, K. C., "Investigation of Plasma Afterglows with Applications in Nitrogen", NASA Technical Note D-2226 (1963).

5. Noon, J. H. and E. H. Holt, "Radiation Temperature Measurements of the Nitrogen Afterglow Plasma", Phys. Rev., 150, 121-123 (1966).

6. McLennan, E. A. and L. Goldstein, "Atomic Density Measurements in $\mathrm{N}_{2}, \mathrm{O}_{2}$ and $\mathrm{H}_{2}$ Afterglow Plasmas", AFCRE Report 66-88 (1966).

7. Hurle, E. R., "On the Thermal Energy Transfer Between Free Electrons and Molecular Vibration", J. Chem. Phys., 4I, 3592-3603 (1966).

8. van Lint, V. A. J., "Ionization Afterglow Measurements on Nitrogen", IEEE Trans., NS-11, 266-271 (1964).

9. Patel, C. K. N., "Selective Excitation through Vibrational Energy Transfer and Optical Maser Action in $\mathrm{N}_{2}-\mathrm{CO}_{2}{ }^{\prime}$, Phys. Rev. Letters, 13, 617-619 (1964).

10. Morgan, J. E. and H. I. Schiff, "The Study of Vibrationally Excited $\mathrm{N}_{2}$ Molecules with the Aid of an Isothermal Calorimeter", Can. J. Chem., 41, 903-912 (1963).

11. Anderson, J. M. and L. Goldstein, "Interaction of EM Waves of Radio-frequency in Isothermal Plasmas", Phys. Rev., 100, 1037-1046 (1955).

12. Phelps, A. V., O. T. Fundingsland and S. C. Brown, "Microwave Determination of the Probability of Collision of Slow Electrons in Gases," Phys. Rev., 84, 559-562 (1951).

13. Formato, D. and A. Gilardini, "Microwave Determinations of Afterglow Temperatures and Electron Collision Frequencies in Nitrogen", Ionization Phenomena in Gases (Proc. of 4th International Conf.), Vol. 1, 99-104, North Holland (1960).

14. Engelhardt, A. G., A. V. Phelps and C. G. Risk, "Determination of Momentum Transfer and Inelastic Collision Cross-sections for Electrons in Nitrogen Using Transport Coefficients", Phys. Rev., 135, A 1566-1574 (1964).

15. Ajello, J., "The Probability of Collision for Momentum Transfer of Slow Electrons in a Nitrogen Plasma", NASA Contractor Report, CR 104 (1964). 
16. Fields, H., G. Bekefi and S. C. Brown, "Microwave Emission from NonMaxweliian Plasmas", Phys. Rev., 129, 506-515 (1963).

17. Balszuk, P. R., J. H. Noon and E. H. Holt, "Non-Maxwellian Form of the Electron Velocity Distribution in the Nitrogen Afterglow Plasma", J. Appl. Phys. (to be published).

18. Haskell, R. E. and E. H. Holt, "Representation of Propagation Parameters for the Plasma in a Magnetic Field", Proc. of IRE, 49, 1584 (1961).

19. Haskell, R. E. and E. H. Holt, "Study of Electromagnetic Wave Polarization in Magneto-Plasmas by a Matrix Method of Crystal Optics", Proc. of the IRE 49, $1584-1585$ (1961).

20. Haskell, R. E., "Polarization Transforming Properties of Anisotropic Plasmas", Rensselaer Polytechnic Institute, Plasma Research Laboratory, TR8 (1963).

21. Rudzki, J. E. and E. H. Holt, "Ten-Kilogauss, Air-Cooled Magnet for Plasma Research", Rev. Sci. Instr., 34, II55-II56 (1963).

22. Allen, P. J. and R. D. Tompkins, "An Instantaneous Microwave Polarimeter", Proc. of the IRE, 47, 1231-2137 (1959).

23. Bitzer, R. A. and E. H. Holt, "A Microwave Lens System for Plasma Diagnostics", NASA Contractor Report, CR-319 (1965).

24. Van Buskirk, L. F. and C. I. Hendrix, "The Zone Plate as a Radio-Trequency Focusing Element", IRE Trans., AP 9, 319-320 (1961).

25. Huchital, D. A., J. F. Reynolds, and E. H. Folt, "Characteristics of the Kadomtsev Instability", AIAA paper 66-157.

26. Huchital, D. A. and E. H. Holt, "Modes of the Kadomtsev Instability", Phys. Rev. Letters, 16, 677-680 (1966).

27. Kadomtsev, B. B., "Convection of the Plasma of a Positive Column in a Magnetic Field", Sov. Phys. - Tech. Phys., 6, 927-933 (1962).

28. Huchital, D. A. and E. H. Holt, "Sub-Threshold Study of the Azimuthal Modes of the Kadomtsev Plasma Instability", Appl. Phys. Letters, 8 , 321-322 (1966).

29. Huchital, D. A. and E. H. Holt, "Transport Phenomena in Ionized Gases as an Initial Value Problem", Phenomena in Ionized Gases (Proc. 7th International Conf.), B. Perovic and D. Tosic, eds., Vol. 2, 95-99, Gradevinska Knijiga Publishing House, Beograd, (1965) and NASA Contractor Report CR-559 (1965).

30. Kajima, S. and S. Hagiwara, "Radiation of Plasma Oscillations", J. Phys. Soc. Japan, 15, 1904 (1960). 
31. Demirkhanov, R. A., A. K. Gevorkov and A. F. Popov, "Interaction of a Beam of Charged Particles with a Plasma", Sov. Phys. - Tech. Phys., 5, 290-294 (1960).

32. Looney, D. H. and S. C. Brown, "The Excitation of Plasma Oscillations", Phys. Rev., 93, 965-969 (1954).

33. Cohen, M. H., "Radiation in a Plasma. I. Cerenkov Effect", Phys. Rev., 123, 711-721 (1961).

34. Kharchenko, I. F., Y. B. Fainberg, R. W. Nikolaev, E. A. Kornilov, E. A. Lutsenko and N. S. Pedenko, "Interaction of an Electron Beam with a Plasma", Sov. Phys. JETP, 11, 493-498 (I960).

35. Allen, M. A. and G. S. Kino, "Interaction of an Electron Beam with a Fully Ionized Plasma", Phys. Rev. Letters, 6, 163-165 (1961).

36. Gaerttner, E. R., M. L. Yeater and R. R. Fullwood, "Operating Experience with the RPI Linac", IRE Trans. Nucl. Sic., NS-9, 23-26 (1962).

37. Mendell, J. S. and E. H. Holt, "Search for Plasma Oscillations Stimulated by a Prc-Bunched Electron Beam from the Rensselaer Linear Accelerator", NASA Contractor Report CR-819 (1967). 